\title{
Comparative genomic analysis of Vibrio parahaemolyticus: serotype conversion and virulence
}

Yuansha Chen ${ }^{1,2}$, O Colin Stine ${ }^{3}$, Jonathan H Badger ${ }^{7}$, Ana I Gil ${ }^{4}, G$ Balakrish Nair ${ }^{5}$, Mitsuaki Nishibuchi ${ }^{6}$ and Derrick E Fouts ${ }^{7 *}$

\begin{abstract}
Background: Vibrio parahaemolyticus is a common cause of foodborne disease. Beginning in 1996, a more virulent strain having serotype 03:K6 caused major outbreaks in India and other parts of the world, resulting in the emergence of a pandemic. Other serovariants of this strain emerged during its dissemination and together with the original O3:K6 were termed strains of the pandemic clone. Two genomes, one of this virulent strain and one pre-pandemic strain have been sequenced. We sequenced four additional genomes of $V$. parahaemolyticus in this study that were isolated from different geographical regions and time points. Comparative genomic analyses of six strains of $V$. parahaemolyticus isolated from Asia and Peru were performed in order to advance knowledge concerning the evolution of $V$. parahaemolyticus; specifically, the genetic changes contributing to serotype conversion and virulence. Two pre-pandemic strains and three pandemic strains, isolated from different geographical regions, were serotype O3:K6 and either toxin profiles (tdh+, trh-) or (tdh-, trh+). The sixth pandemic strain sequenced in this study was serotype O4:K68.

Results: Genomic analyses revealed that the trh+ and $t d h+$ strains had different types of pathogenicity islands and mobile elements as well as major structural differences between the tdh pathogenicity islands of the pre-pandemic and pandemic strains. In addition, the results of single nucleotide polymorphism (SNP) analysis showed that 94\% of the SNPs between O3:K6 and O4:K68 pandemic isolates were within a $141 \mathrm{~kb}$ region surrounding the O- and Kantigen-encoding gene clusters. The "core" genes of $V$. parahaemolyticus were also compared to those of $V$. cholerae and $V$. vulnificus, in order to delineate differences between these three pathogenic species. Approximately one-half (49-59\%) of each species' core genes were conserved in all three species, and $14-24 \%$ of the core genes were species-specific and in different functional categories.

Conclusions: Our data support the idea that the pandemic strains are closely related and that recent South American outbreaks of foodborne disease caused by $V$. parahaemolyticus are closely linked to outbreaks in India. Serotype conversion from O3:K6 to O4:K68 was likely due to a recombination event involving a region much larger than the O-antigen- and K-antigen-encoding gene clusters. Major differences between pathogenicity islands and mobile elements are also likely driving the evolution of $V$. parahaemolyticus. In addition, our analyses categorized genes that may be useful in differentiating pathogenic Vibrios at the species level.
\end{abstract}

\footnotetext{
* Correspondence: dfouts@jcvi.org

${ }^{7}$ The J. Craig Venter Institute, Rockville, MD, USA

Full list of author information is available at the end of the article
} 


\section{Background}

Vibrio parahaemolyticus is a halophilic bacterium which has long been recognized [1] as a human pathogen that causes gastroenteritis and, occasionally, wound infections and sepsis in immunocompromised patients. It is the leading etiologic agent for bacterial foodborne disease in Japan and other parts of Asia, and it is the most common bacterial cause of seafoodassociated disease in the United States. Prior to 1996, there was no specific serotype of $V$. parahaemolyticus that was associated with disease outbreaks, and the bacterium had never been reported to cause a pandemic. However, during that year, a major outbreak occurred in India, $>50 \%$ of the $V$. parahaemolyticus strains isolated from patients were serotype O3:K6 [2]. Also, the outbreak rapidly spread to other countries in Asia, South America, North America, Africa and Europe, resulting in a pandemic affecting tens of thousands of people $[2,3]$. During its global dissemination, $>20$ serovariants (including O3:K6, O4:K68, O1:K25, O1:KUT [untypable], and others [2,4,5] rapidly evolved from the original pandemic O3:K6 strain. The pandemic O3:K6 and its serovariants are termed strains of the pandemic clone.

A thermostable direct hemolysin (TDH) is recognized [6] as the most important virulence factor of $V$. parahaemolyticus, and a TDH-related hemolysin (TRH) is believed to account for the virulence of strains that do not produce TDH. Prior whole-genome sequencing $[7,8]$ of a serotype O3:K6, pandemic isolate designated RIMD2210633 identified two type III secretion systems (T3SS). T3SSI is present in all $V$. parahaemolyticus isolates examined and is required for the bacterium's cytolytic activity [8]; whereas, T3SSII is required for enterotoxicity and is located in the $t d h$-containing pathogenicity island $[7,8]$.

Outbreaks of diarrheal disease caused by $V$. parahaemolyticus may pose a significant health threat. Thus far, the most affected country (other than India) has been Chile, where $>10,000$ cases were reported during
2005. This observation suggests that, under appropriate conditions, $V$. parahaemolyticus may cause large-scale outbreaks comparable to those elicited by $V$. cholerae. At the present time, the reasons for the pandemic strains' rapid increase in virulence/prevalence have not been rigorously determined. In addition, the mechanism(s) for rapid serotype conversion warrant further study. Furthermore, it is not clear whether the virulence mechanisms of $t d h+$ and $t r h+$ strains are similar. Therefore, in order to address some of these questions, we performed rigorous genomic analyses of two pre-pandemic and four pandemic isolates of $V$. parahaemolyticus.

\section{Results and Discussion}

\section{Comparative genomics of $V$. parahaemolyticus}

Prior to this study, an O3:K6 pandemic isolate (strain RIMD2210633) was sequenced to completion [7] and an O3:K6 non-pandemic isolate (strain AQ3810) was sequenced to draft status [9]. In this study, we sequenced four additional isolates of $V$. parahaemolyticus to at least 8-fold draft coverage, for a total of six clinical isolates; two non-pandemic and four pandemic (Table 1). The two non-pandemic strains, AQ3810 and AQ4037, were isolated in 1983 and 1985, respectively, and both originated from Southeast Asia. Throughout the remainder of this study, we will refer to these two non-pandemic isolates as "pre-pandemic" because they were isolated prior to the documented start of the pandemic. Three of the pandemic isolates were from Southeast Asia, including strain RIMD2210633 in 1996, strain AN5034 in 1998, and strain K5030 in 2005, while the fourth pandemic isolate (strain Peru466) was isolated from Peru in 1996. Therefore, the isolates represented two geographic areas where major outbreaks occurred. In addition, they also have different serotypes and toxin profiles. All of the pandemic strains were $(t d h+t r h-)$ and the pre-pandemic strains were either $(t d h+t r h-)$ or $(t d h-t r h+)$, thus representing two potentially different virulence mechanisms. To improve our understanding

Table 1 Six $V$. parahaemolyticus strains analyzed during this study

\begin{tabular}{|c|c|c|c|c|c|c|c|c|c|}
\hline Year & Strain & Source & Serotype & $t d h$ & trh & \# contigs & Contig N50 ${ }^{+\S}$ (bp) & Max. Contig & Reference \\
\hline $1983^{\ddagger}$ & AQ3810 & Singapore & O3:K6 & + & - & 1037 & 52609 & 295134 & [9] \\
\hline $1985^{\ddagger}$ & AQ4037 & Maldives & O3:K6 & - & + & 164 & 67710 & 241746 & This study \\
\hline 1996 & RIMD2210633 & Thailand & O3:K6 & + & - & 2 & N.A. & 3288558 & [7] \\
\hline 1996 & Peru466 & Peru & O3:K6 & + & - & 149 & 81497 & 273858 & This study \\
\hline 1998 & AN5034 & Bangladesh & O4:K68 & + & - & 54 & 346246 & 1183081 & This study \\
\hline 2005 & K5030 & India & O3:K6 & + & - & 164 & 62978 & 657114 & This study \\
\hline
\end{tabular}

${ }^{\dagger}$ The contig N50 is the length of the smallest contig in the set that contains the fewest (largest) contigs whose combined length represents at least $50 \%$ of the assembly [41].

${ }^{\S}$ The contig N50 was calculated from the Celera assembly, not the contigs submitted to Genbank.

‡Pre-pandemic years.

N.A. Not Applicable. 
of the pandemic clone's evolution during their global dissemination, the genome of a Peruvian isolate (strain Peru466) [10] was sequenced and compared to the genomes of Asian isolates collected at different time points during the pandemic. In the later stage of the pandemic, there were fewer cases of infection in South Asia; thus, $V$. parahaemolyticus isolated during this time seems to be less virulent (Nair, personal observation). Therefore, an isolate (strain K5030) collected in 2005 from India was included and considered a "less virulent" late stage pandemic isolate in this study. Also, the genome of a never-before-sequenced serotype O4:K68 pandemic isolate (strain AN5034) was characterized in order to advance our understanding of the mechanism for its serotype conversion.

The pan genome of the six $V$. parahaemolyticus strains we examined had 6,616 chromosomal coding genes, and each individual genome (excluding plasmids) had an average of 4,673 coding genes (Figure 1). Three thousand twenty eight genes, ca. $71 \%$ of the coding genes were present in all the strains (Additional file 1). However, that number may be lower than the actual number because the genomes, except for the genome of RIMD2210633, were not sequenced to completion. Therefore, some of the open-reading frames (ORFs) that bordered contigs may have failed to meet the cut-offs and, subsequently, were treated as not present. The four newly sequenced genomes displayed a high degree of synteny with RIMD2210633 (Figure 1). There was very little rearrangement of the genome of the pre-pandemic strain AQ4037 and essentially no rearrangement in the pandemic strains. Because the gaps are not closed for five of the genomes, our report of synteny represents our best estimation.

\section{Super integron}

V. parahaemolyticus harbors a super integron (SI) on chromosome I. The SI is about $48 \mathrm{~kb}$ long and contains ca. 77 ORFs, which is much smaller than the SIs in $V$. cholerae $(120 \mathrm{~kb})$ and in $V$. vulnificus $(138 \mathrm{~kb})$. Most of the ORFs in the SI regions encode hypothetical proteins. The SI integrases were identical in the six $V$. parahaemolyticus strains examined, but the cassettes in the SI regions of the pre-pandemic strains varied greatly from those of the pandemic strains. For example, only 24 and 28 of the 77 ORFs in the pandemic strains' SI regions were present in those of pre-pandemic strains AQ3810 and AQ4037, respectively. However, the cassettes in the SI regions of the four pandemic strains were nearly identical; i.e., they contained only a few point mutations. The only exception was isolate K5030, which had an additional six hypothetical proteins inserted between the integrase and the rest of the cassettes. These observations indicate that the integrase is active in $V$. parahaemolyticus and contributes to species evolution. However, the fact that its SI region is smaller than those of other pathogenic Vibrio species, and the presence of highly conserved cassettes in the pandemic strains, suggests that the genomes of $V$. parahaemolyticus may be more stable than those of other pathogenic Vibrios.

\section{Pathogenicity islands, prophages, and integrated elements}

Only the pandemic strains examined in this study contained the pathogenicity islands previously described [7] for $V$. parahaemolyticus (Table 2). In addition, we detected various prophages and integrated elements using Phage_Finder [11]. Prophage f237, which has been widely used as a genetic marker for the pandemic clone [12], was present in chromosome I (loci VP1549-1562 in strain RIMD2210633) of all the pandemic strains we examined, but it was absent from the pre-pandemic strains (Figure 2A). However, a prophage similar to f237 was present in pre-pandemic strain AQ4037, in the same location occupied by $\mathrm{f} 237$ in the pandemic strains (Figure 2A). In addition to $\mathrm{f} 237$, another prophage was identified adjacent to f237 (loci VP1563-1586 in strain RIMD2210633) in all of the pandemic strains and in pre-pandemic strain AQ3810, but it was absent from strain AQ4037. Also, a second copy of that prophage was present in chromosome II of the pandemic strains and in strain AQ3810 (Figure 2B). In addition, a prophage region unique to the serotype $\mathrm{O} 4: \mathrm{K} 68$, pandemic strain AN5034 (AN5034_0425-0489) was identified by Phage_Finder (Table 2 and Additional file 1).

Each of the strains we studied had one or two integrated elements targeting the tmRNA gene (Table 2). For example, Peru466, AN5034, and K5030 had two different, integrated elements inserted in tandem into the 3' end of their tmRNA genes. The element closest to the tmRNA gene contained two genes that may influence virulence: a putative cyclic diguanylate phosphodiesterase EAL domain protein and an AraC superfamily putative fimbrial transcriptional activator. The second element was distinguished by the presence of a ribonuclease $\mathrm{H}$-encoding gene. The first element was present in strains AQ3810 and AQ4037, but not in strain RIMD2210633. However, strains AQ3810 and AQ4037 lacked the ribonuclease $\mathrm{H}$-encoding element present in strain RIMD2210633.

\section{Characterization of the pathogenicity islands}

Pre-pandemic strain AQ4037 is tdh-, trh+ and ureasepositive, and its genome sequence revealed a pathogenicity island (hereafter called trhPAI) containing 81 ORFs (Figure 3). Another pathogenicity island (hereafter referred to as $t d h \mathrm{PAI}$ ) was previously identified in 
A.
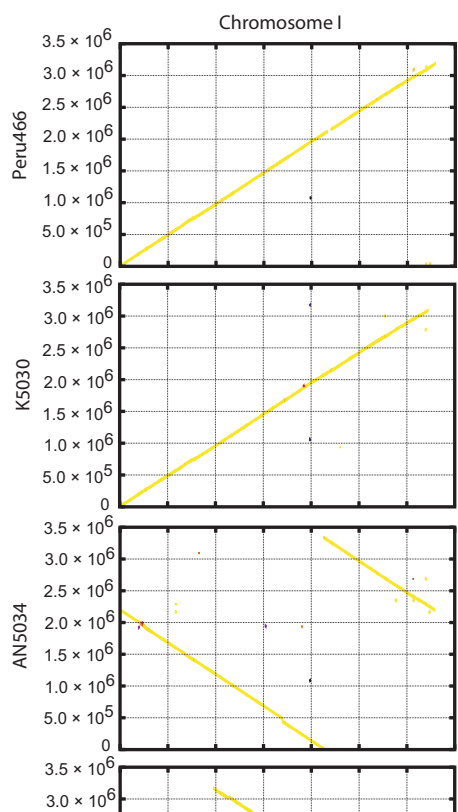

$3.5 \times 10^{6}$
$-2.0 \times 10^{6}$

角 $2.0 \times 10^{6}$.

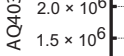

$1.0 \times 10^{6}$

$1.0 \times 10^{6}$
$5.0 \times 10^{5}$

$\times 10^{5}$

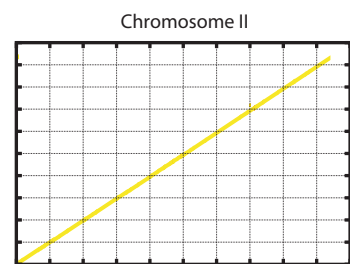

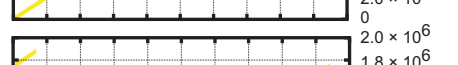

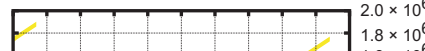

$1.6 \times 10^{6}$

$1.4 \times 10^{6}$

$1.2 \times 10^{6}$

$1.0 \times 10^{6}$

$8.0 \times 10^{5}$

$6.0 \times 10^{5}$

$4.0 \times 10^{5}$

$2.0 \times 10^{5}$

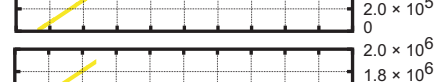

$1.8 \times 10^{6}$
$1.6 \times 10^{6}$

$1.4 \times 10^{6}$

$1.4 \times 10^{6}$

$1.2 \times 10^{6}$

$8.0 \times 10^{5}$

$8.0 \times 10^{5}$
$6.0 \times 10^{5}$

$4.0 \times 10^{5}$

$4.0 \times 10^{5}$
$2.0 \times 10^{5}$
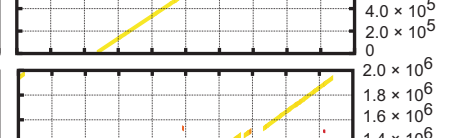

$1.6 \times 10^{6}$
$1.4 \times 10^{6}$

$1.2 \times 10^{6}$

$1.0 \times 10^{6}$

$8.0 \times 10^{5}$

$6.0 \times 10^{5}$

$6.0 \times 10^{5}$

$4.0 \times 10^{5}$

잉

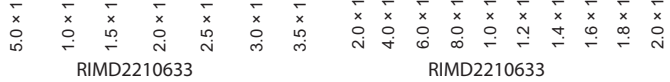

B.

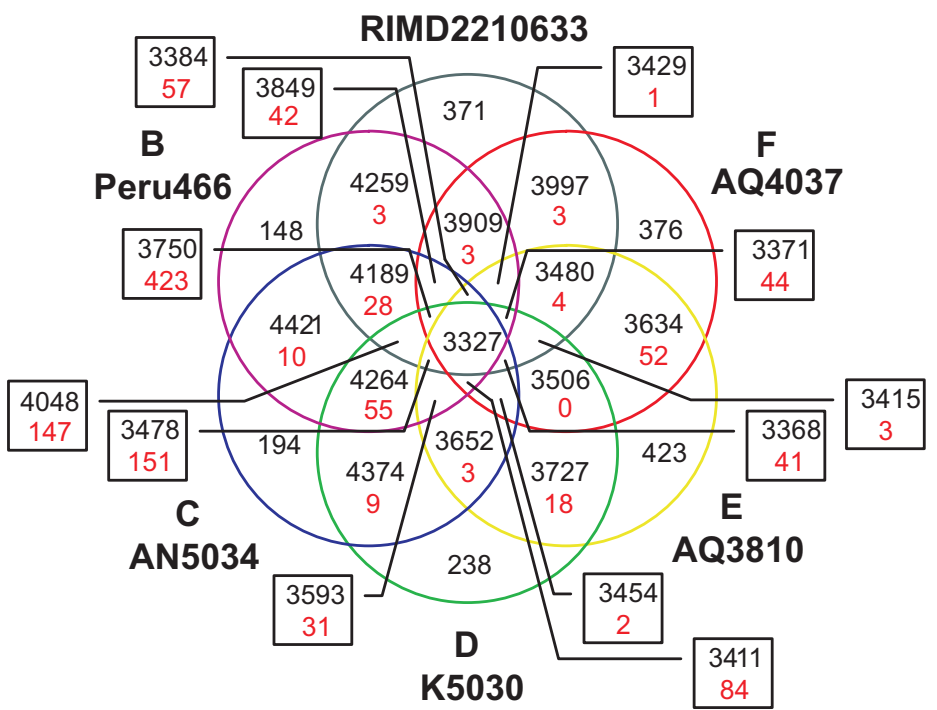

Figure 1 Whole-genome comparison of six V. parahaemolyticus strains. Panel A: Colored lines denote the percent identities of protein translations, and they are plotted according to their locations in the reference strain (RIMD2210633) and query strain's genomes. Panel B: Venn diagrams indicate the number of shared proteins (black) and unique proteins (red) within a particular relationship for all six $V$. parahaemolyticus strains. 
Table 2 Variable regions in V. parahaemolyticus

\begin{tabular}{|c|c|c|c|c|c|c|c|c|c|}
\hline \# & $\begin{array}{l}\text { Region or insertion site } \\
\text { relative to RIMD2210633 }\end{array}$ & $\begin{array}{l}\text { Number } \\
\text { of ORFs }\end{array}$ & Function & $\begin{array}{l}\text { RIMD } \\
\text { (O3:K6 } \\
1996) \\
\end{array}$ & $\begin{array}{l}\text { Peru466 } \\
\text { (O3:K6 } \\
1996) \\
\end{array}$ & $\begin{array}{l}\text { AN5034 } \\
(04: K 68 \\
1998)\end{array}$ & $\begin{array}{l}\text { K5030 } \\
(\mathrm{O}: \mathrm{K} 6 \\
2005) \\
\end{array}$ & $\begin{array}{l}\text { AQ3810 } \\
(03: K 6 \\
1983) \\
\end{array}$ & $\begin{array}{l}\text { AQ4037 } \\
\text { (O3:K6 } \\
1985) \\
\end{array}$ \\
\hline 1 & $\begin{array}{l}\text { Between VP0001-0002 } \\
\text { (K5030_3039-3061) }\end{array}$ & 23 & $\begin{array}{l}\text { DNA sulfur } \\
\text { modification proteins }\end{array}$ & - & - & - & + & - & - \\
\hline 2 & VP0197-0238 & 42 & O3:K6 LPS/CPS & + & + & - & + & + & + \\
\hline 3 & $\begin{array}{l}\text { Replaced VP0197-0238 } \\
\text { (AN5034_1849-1901) }\end{array}$ & 53 & O4:K68 LPS/CPS & - & - & + & - & - & - \\
\hline 4 & $\begin{array}{l}\text { Between VP0248-0249 } \\
\text { (AN5034_1830-1837) }\end{array}$ & 8 & Unknown & - & - & + & - & - & - \\
\hline 5 & VP0380-0403 & 24 & $\begin{array}{l}\text { Type I restriction } \\
\text { endonuclease in tRNA- } \\
\text { Met-1 }\end{array}$ & + & + & + & + & - & - \\
\hline 6 & VP0637-0643 & 7 & $\begin{array}{l}\text { Integrated element } \\
\text { target tmRNA }\end{array}$ & + & + & + & + & - & - \\
\hline 7 & $\begin{array}{l}\text { Between VP0643-0644 } \\
\text { (AN5034_1437-1442) }\end{array}$ & 6 & $\begin{array}{l}\text { Integrated element } \\
\text { target tmRNA }\end{array}$ & - & + & + & + & + & + \\
\hline 8 & VP1071-1076 & 6 & $\begin{array}{l}\text { Unknown, contains } \\
\text { phage integrase }\end{array}$ & + & + & + & + & - & - \\
\hline 9 & VP1077-1087 & 11 & $\begin{array}{l}\text { Unknown, contains } \\
\text { phage integrase }\end{array}$ & + & + & + & + & + & - \\
\hline 10 & VP1385-1421 & 37 & $\begin{array}{l}\text { Type VI secretion } \\
\text { system }\end{array}$ & + & + & + & + & - & + \\
\hline 11 & VP1549-1562 & 14 & Phage f237 & + & + & + & + & - & - \\
\hline 12 & $\begin{array}{l}\text { Replaced VP1549-1562 } \\
\text { (AQ4037_2432-2444) }\end{array}$ & 13 & Phage similar to f237 & - & - & - & - & - & + \\
\hline 13 & VP1563-1586 & 24 & Phage alpha* & + & + & + & + & + & - \\
\hline 14 & $\begin{array}{l}\text { Between VP1604-1605 } \\
\text { (AN5034_0425-0489) }\end{array}$ & 65 & Phage & - & - & + & - & - & - \\
\hline 15 & VP1787-1865 & 78 & Super integron & + & + & + & + & $v$ & $\mathrm{v}$ \\
\hline 16 & $\begin{array}{l}\text { Between VP1864-1865 } \\
\text { (K5030 1808-1814) }\end{array}$ & 7 & $\begin{array}{l}\text { Addition to super } \\
\text { integron, next to } \\
\text { integrase }\end{array}$ & - & - & - & + & - & - \\
\hline 17 & VP1884-1891 & 8 & Unknown & + & + & + & + & - & - \\
\hline 18 & VP1969-1974 & 6 & $\begin{array}{l}\text { Fatty acid and amino } \\
\text { acid metabolism }\end{array}$ & + & + & + & + & - & + \\
\hline 19 & VP2131-2144 & 14 & Hypothetical proteins & + & + & + & + & - & - \\
\hline 20 & $\begin{array}{l}\text { Between VP2275-2276 } \\
\text { (AQ4037_1749-1829) }\end{array}$ & 81 & trh pathogenicity island & - & - & - & - & - & + \\
\hline 21 & $\begin{array}{l}\text { Between VP2638-2639 } \\
\text { (AQ4037_1361_1383) }\end{array}$ & 23 & $\begin{array}{l}\text { Hypothetical proteins, } \\
\text { contains integrase }\end{array}$ & - & - & - & - & - & + \\
\hline 22 & VP2900-2910 & 11 & Hypothetical proteins & + & + & + & + & - & - \\
\hline 23 & VPA0434-0440 & 7 & Hypothetical proteins & + & + & + & + & - & - \\
\hline 24 & VPA0889-0912 & 24 & Phage beta* & + & + & + & + & + & - \\
\hline 25 & VPA1254-1270 & 17 & Unknown & + & + & + & + & - & - \\
\hline 26 & VPA1310-1398 & 86 & $\begin{array}{l}\text { tdh pathogenicity } \\
\text { island }\end{array}$ & + & + & + & + & + & - \\
\hline 27 & $\begin{array}{l}\text { Replaced VPA1310-1313 } \\
\text { (AN5034_A0845-A0851) } \\
\end{array}$ & 7 & Hypothetical proteins & - & + & + & + & - & - \\
\hline 28 & $\begin{array}{l}\text { Replaced VPA1310-1398 } \\
\text { (AQ4037_A1228-A1253) }\end{array}$ & 25 & $\begin{array}{l}\text { Nutrient uptake and } \\
\text { metabolism }\end{array}$ & - & - & - & - & + & + \\
\hline
\end{tabular}

In the first column, if genes are absent from RIMD, the gene numbers in one of the other genomes are indicated in the parenthesis.

* These two phages are very similar.

$\checkmark$ Variable contents in the super integron 


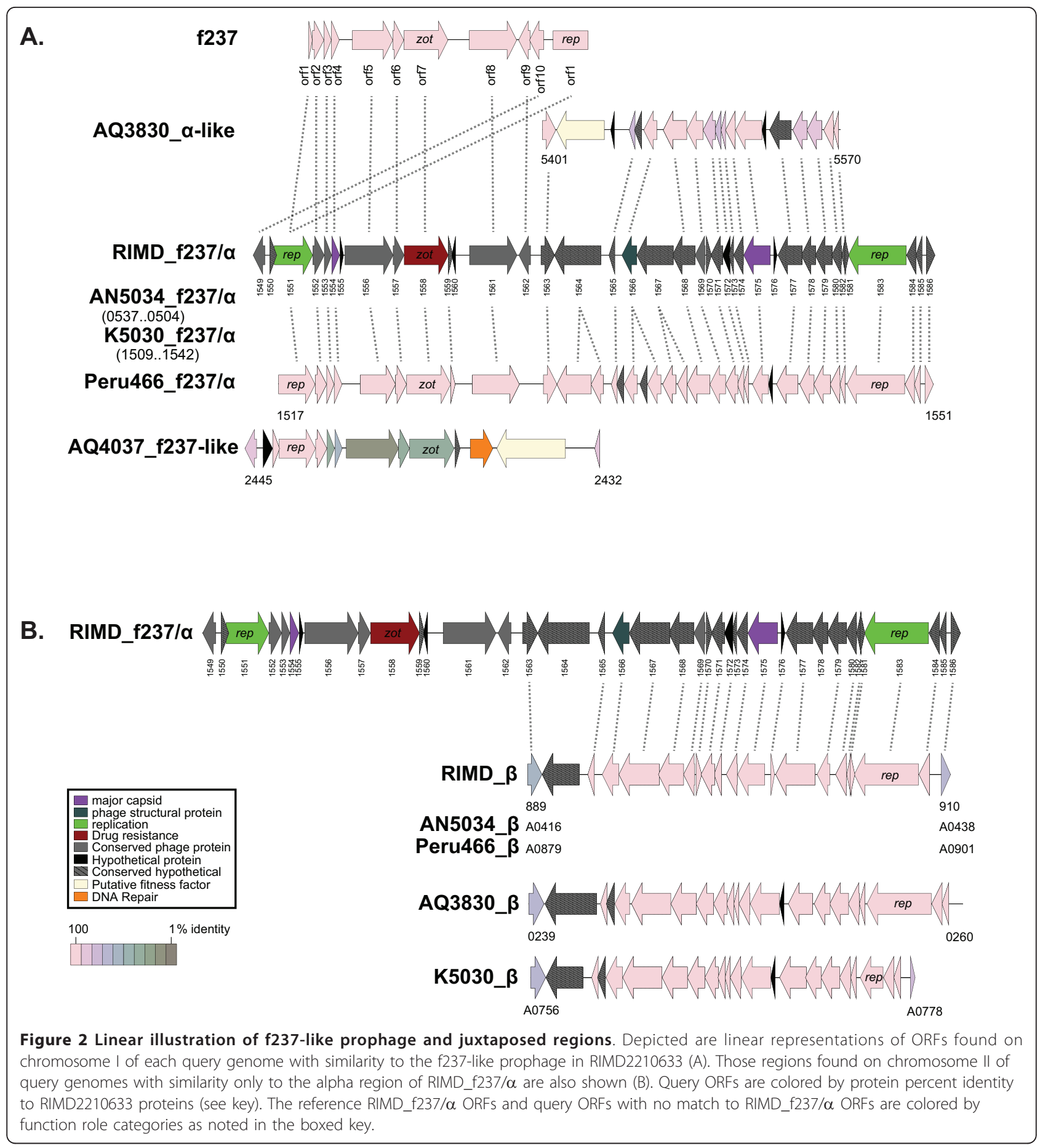

chromosome II of pandemic strain RIMD2210633 and includes loci VPA1310-1398 (Figure 3). tdhPAI contains a type III secretion system (T3SSII) and two copies of $t d h$; whereas, trhPAI contains trh, an integrase, transposases, a urease gene cluster, a peptide/nickel transportation system, and a T3SS that is different from the one in tdhPAI (Figure 3). The T3SS in AQ4037's trhPAI is similar to T3SSII $\beta$ in $V$. parahaemolyticus TH3996, which is related to the T3SS in non-O1, non-O139 strains of $V$. cholerae [13]. Interestingly, trhPAI was found in chromosome II of strain TH3996, but it was located in chromosome I of strain AQ4037. This discrepancy in chromosomal location may be providing a clue to the pathogenicity island's mobility.

Close examination of the tdhPAI region in the six genomes revealed major differences between the 


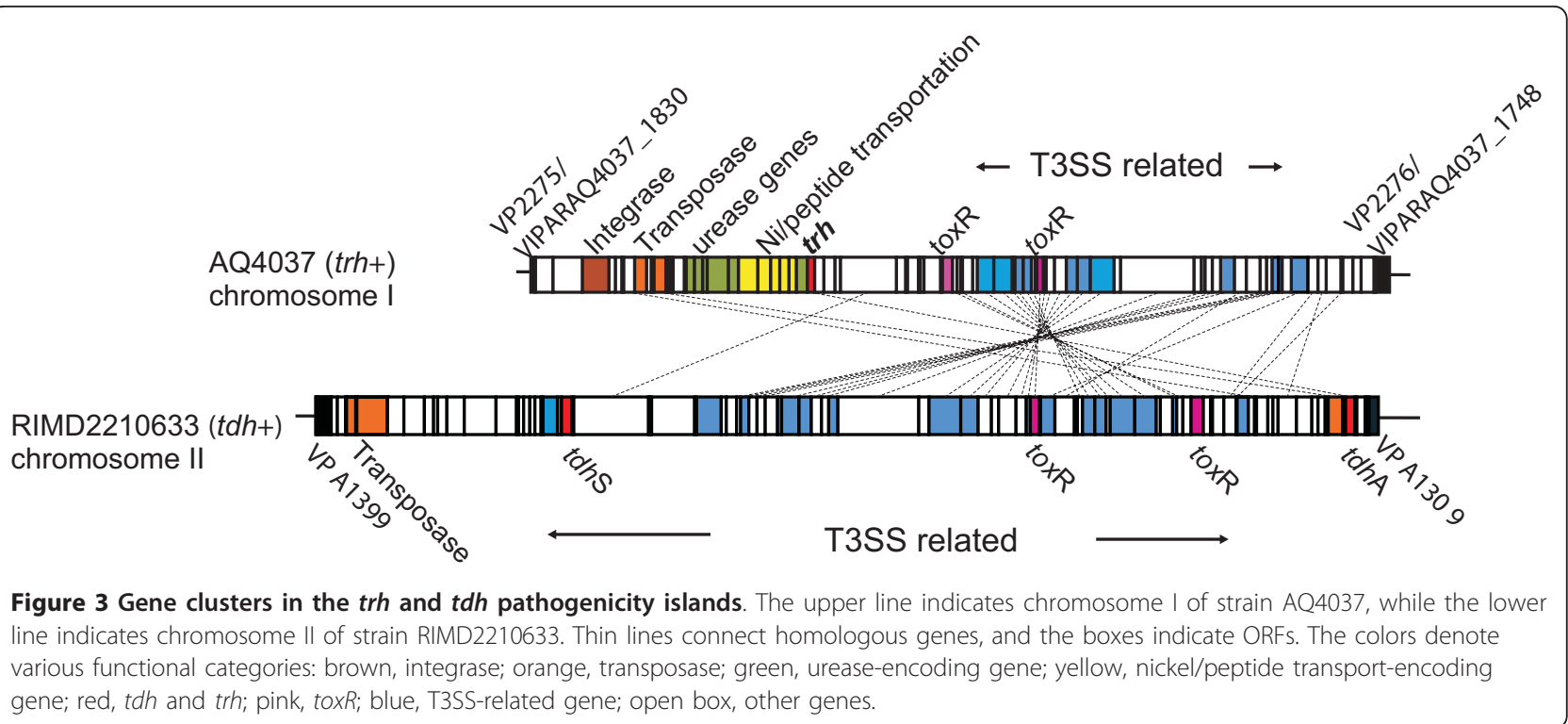

pre-pandemic and pandemic strains (Figure 4). The four epidemic strains' $t d h$ PAIs were very similar to one another; however, the entire pathogenicity island was absent from the pre-pandemic, tdh-strain AQ4037. Instead, that strain contained a pre-pandemic-specific region of 18 ORFs important for the uptake and metabolism of carbon sources and other nutrients. In addition, although pre-pandemic strain AQ3810 contained both $t d h$ PAI and the pre-pandemic nutrient uptake region, it had an inverted $t d h \mathrm{~S}$ gene (Figure 4). Thus, the pre-pandemic and pandemic strains exhibited major differences between the region upstream of the pathogenicity island and in $t d h$ 's orientation. Whether those variations affect the expression of the pathogenicity island's genes, which contributes to differences between the pre-pandemic and pandemic strains' virulence, remains to be determined. The pathogenicity island's organization suggests that an ancestral strain possessing the O3:K6 serotype may have recruited a $t$ dhPAI next to VPA1309, which yielded a transient strain that subsequently lost the pre-pandemic island and gave rise to the pandemic strains. Another possibility is that the $t d h+$, pre-pandemic strain and the pandemic strains independently recruited the pathogenicity islands into the same location.

The $t d h$ and $t r h$ genes are related but vary substantially [14], and many variants of $t d h$ and two trh genes have been described. They have been found in various Vibrio species and their phylogenetic relationships are not in accordance with the relationship of the host species [14]. Although most of them are in the chromosomes, some of them are present in plasmids, a finding that is consistent with their proposed mobility. Also, the presence of $t r h$ and $t d h$ on both chromosomes of $V$. parahaemolyticus supports the idea that they may have been acquired by lateral gene transfer and may have integrated into the bacterium's genome during independent events. Also, since V. parahaemolyticus strains that have both $t d h$ and trh have been described [15], it might be worthwhile to sequence those strains'

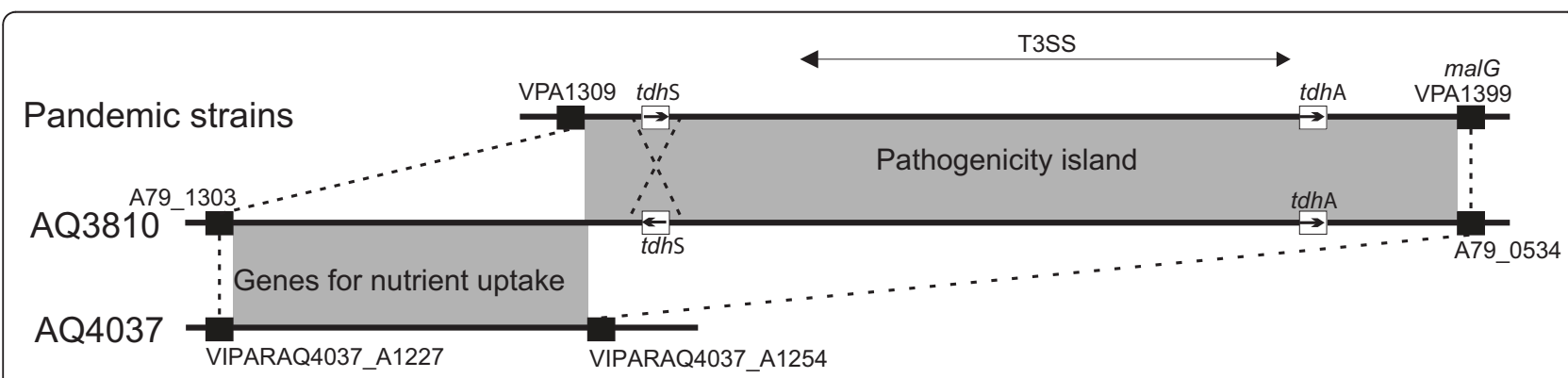

Figure 4 Diagram of the $\boldsymbol{t} \boldsymbol{d} \boldsymbol{h}$ pathogenicity island. Solid black boxes indicate the common border genes, and the homologous genes are connected by dotted lines. Homologous regions are shaded and tdh genes are indicated with arrows showing the direction of transcription. Other ORFs are omitted for simplicity. 
genomes in order to understand the evolutionary history of $t d h$ and trh in their hosts.

TDH and TRH are the only confirmed virulence factors of $V$. parahaemolyticus; however, their precise roles are not well understood. Genomic sequencing revealed that the genes encoding those toxins are in close proximity to a T3SSII system, which suggests they may have the same origin as that transport system. Thus, it is tempting to speculate that TDH (and/or TRH) and T3SSII have coordinating activities related to the virulence of $V$. parahaemolyticus. Some translocon proteins and effectors have been identified for T3SSII [16,17]; however, the putative relationship between TDH, TRH, and T3SSII needs further investigation. Another T3SS (T3SSI) identified in the $V$. parahaemolyticus genome was demonstrated $[8,18]$ to be required for cytolytic activity. However, T3SSI was conserved in all of the genomes we examined.

\section{Variability within the 03:K6 genetic locus}

Since 1996, serotype O3:K6 has predominated among clinical isolates of $V$. parahaemolyticus, thus that serotype has been associated with the bacterium's increased virulence. However, strains of O3:K6 serotype had been isolated more than a decade before the pandemic initiated. It is not clear if there are variations within the O3:K6 genetic determinants between non-pandemic and pandemic strains, and thus causing subtle structural difference of the $\mathrm{O}$ and $\mathrm{K}$ antigens that could not be detected by the serotyping techniques. Therefore, we examined the $\mathrm{O}$ - and $\mathrm{K}$-antigen-encoding gene clusters in the pre-pandemic and pandemic O3:K6 strains for any variations that may explain the observed increase in virulence. The $\mathrm{O}$ - and $\mathrm{K}$-antigen-encoding gene clusters are juxtaposed in $V$. parahaemolyticus O3:K6 [19]. In strain RIMD2210633, they are located at loci VP01900238 in chromosome I (position 201,797-253,279) $[20,21]$. However, that region was conserved in all O3: K6 strains (sharing $>99.5 \%$ amino acid-encoding identity), which suggests that the serotype of $V$. parahaemolyticus may not be directly related to the pandemic strain's increased virulence.

\section{Serotype conversion from 03:K6 to 04:K68}

In addition to serotype O3:K6, > 20 other serotypes of $V$. parahaemolyticus were detected among pandemic strains [2]. The mechanism for this serotype conversion remains unknown. Since the O- and $\mathrm{K}$-antigen loci are tightly linked on the chromosome [19], a recombination event involving this region would enable rapid conversion of serotypes. Before comparing the $\mathrm{O}$ - and $\mathrm{K}$ antigen region between O3:K6 and O4:K68 serotypes, we first wanted to determine the variability of this region within the O4:K68 serotype as we did above for the O3:K6 serotype. We compared the O- and K- region of strain AN5034 (O4:K68), AN5034_1842-1901, to the $\mathrm{O}$ - and $\mathrm{K}$ - region of another O4:K68 strain designated NIID242-200 [21]. Both clusters were nearly identical (9 mismatches in a 63-kb-long region), as observed above for the O3:K6 loci. When comparing the O- and K-antigen regions between strain AN5034 (O4:K68) and strain RIMD2210633 (O3:K6), this region varied substantially, except for the first seven genes, suggesting recombination as a method for serotype conversion. To help identify the scope of the recombination event, we analyzed the distribution of single-nucleotide polymorphisms (SNPs) in the genome of strain AN5034 compared to strain RIMD2210633. Compared to the other pandemic isolates, strain AN5034 had 2,281 SNPs (excluding insertions and deletions), 2,142 (94\%) of which clustered in a $141 \mathrm{~kb}$ region (position 199,786-341,273 in RIMD2210633 chromosome I, and position 166,252324,726 in AN5034 contig ACFO01000016.1), corresponding to between 2 -kb upstream and 88 -kb downstream of the $\mathrm{O}$ - and $\mathrm{K}$-antigen-encoding gene clusters (Additional file 2 blue highlight, Figure 5 circle 5). This observation suggests that a recombination event involving a much larger region than the $\mathrm{O}$-antigen- and $\mathrm{K}$-antigen loci occurred and gave rise to the new $\mathrm{O} 4$ : K68 serotype during the pandemic.

\section{Origin of the South American outbreaks}

Our results indicated that the pandemic strains are closely related to one another. Strain RIMD2210633 differed from strain $\mathrm{K} 5030$ and the Peruvian strain (Peru466) by 70 and 76 SNPs, respectively (Additional file 2). Peru466 was isolated at approximately the same time the first outbreak (which later evolved into a pandemic) was reported in India. Also, its gene content is almost identical to that of a pandemic Asian strain (RIMD2210633) isolated at the same time, which supports the idea that the pandemic strain spread from Asia to South America very soon after it emerged. Considering the proximity, it is likely that the 2005 outbreak in Chile was caused by strains descended from the Peruvian isolates. We speculate that the strains from South American outbreaks are closely related to the strains from Indian outbreaks. This hypothesis will require genomic sequencing of additional strains from South America, specifically those from Chile, to confirm.

Fewer cases of disease caused by $V$. parahaemolyticus were reported from Asia during the later stages of the pandemic than during its early stages (Nair, personal observation); thus, we suspect that the strains isolated during the later stages were not as virulent as those obtained during its early stages. Therefore, we sequenced and analyzed the genome of strain K5030, 


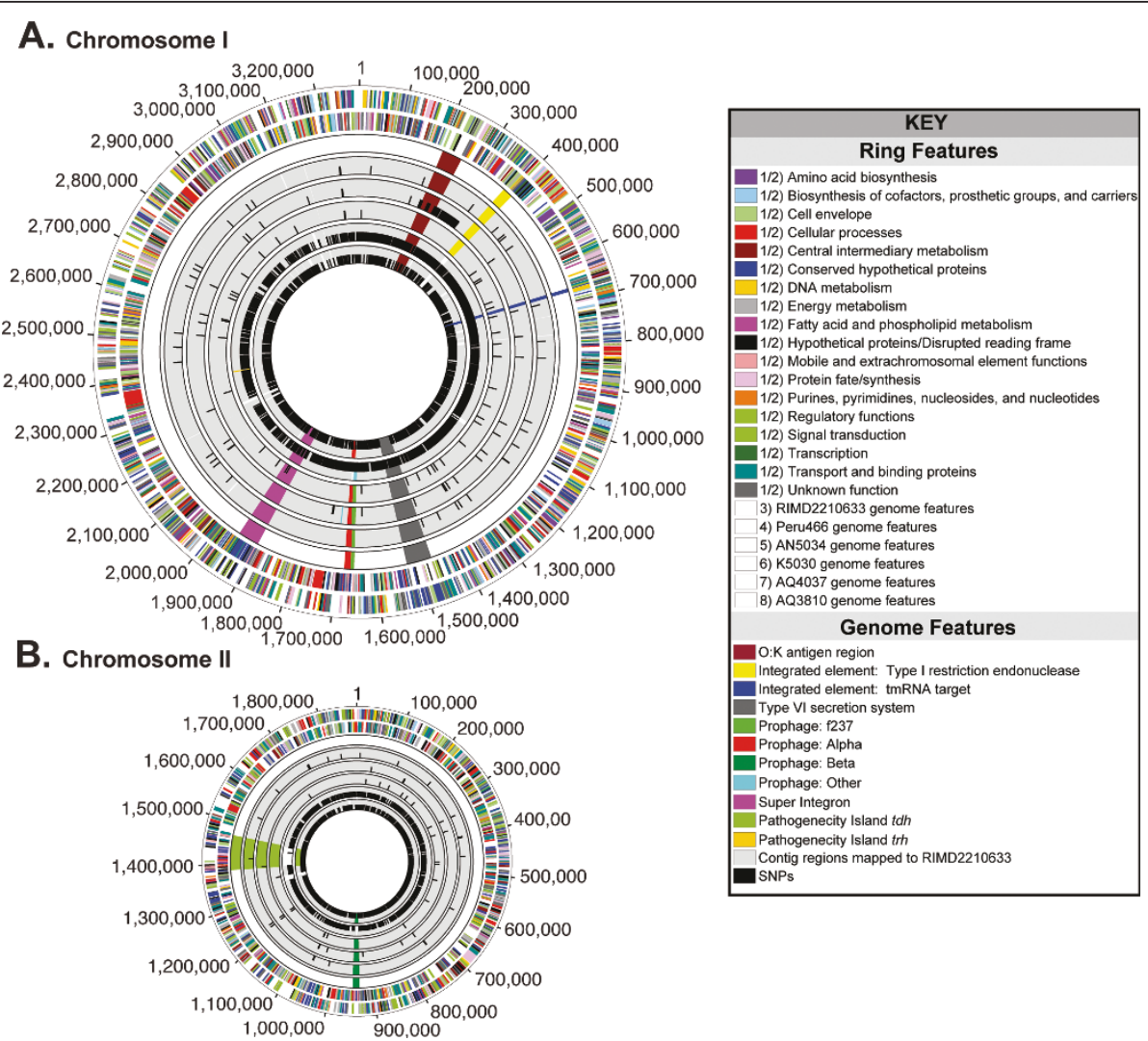

Figure 5 Circular Illustration of Single Nucleotide Polymorphisms and Genome Features Relative to the Reference Strain RIMD2210633. Chromosomes I (A) and II (B) are illustrated as a circles where each concentric circle represents genomic data and is numbered from the outermost to the innermost circle. Circles 1 and 2 represent RIMD2210633 ORFs and are colored based on function role categories. Circle 3 depicts the location in RIMD2210633 of various genomic features described in this study. Circles 4-8 denote the location of SNPs relative to RIMD2210633 and genomic features for strain Peru466, AN5034, K5030, AQ4037, and AQ3810, respectively. Refer to the key for details on color representations and circle numbers.

which was isolated from India almost a decade after the pandemic started, to look for gene deletions and variations. Major gene deletions were not identified in strain K5030; instead, several insertions were detected (Table 2 ). In addition to the cassettes added to the strain's SI, K5030 had an insertion of 23 ORFs (between VP0001 and VP0002) containing a DNA phosphorothioation (dnd) system, which incorporates sulphur into the DNA backbone, $[22,23]$. Recent evidence supports the lateral transfer of dnd genes among bacterial genomes [24]. Besides the dnd genes, there were also two transposases and one integrase in the insertion. However, it is not clear whether the addition of those ORFs reduced the strain's virulence by altering the structure and function of virulence factors.

\section{Comparative genomic analyses of the three major pathogenic Vibrio species}

In order to characterize important genetic differences between $V$. parahaemolyticus, $V$. cholerae and $V$. vulnificus, we compared their core genomes. The $V$. cholerae group consisted of four completely sequenced genomes of toxigenic, serotype O1 strains: N16961, M66-2, MJ-1236 and O395 [25-27]. The V. vulnificus group contained two completely sequenced genomes of strains CMCP6 and YJ016 [28,29], and the V. parahaemolyticus group consisted of strain RIMD2210633 and the two pre-pandemic isolates (strains AQ3810 and AQ4037). The orthologs from each group were extracted by comparing all members of the group, after which the core genes from each group were compared to each other.

Forty-nine to $59 \%$ of the core genes in each species were common to all three species (Figure 6). However, $14-24 \%$ of the core genes in each group were only conserved in its own group and were in different COG (Clusters of Orthologous Genes) categories (Figure 6). These are likely to be the genes that define the bacteria at the species level. Furthermore, each species had specific genes and transporters required for various metabolic 


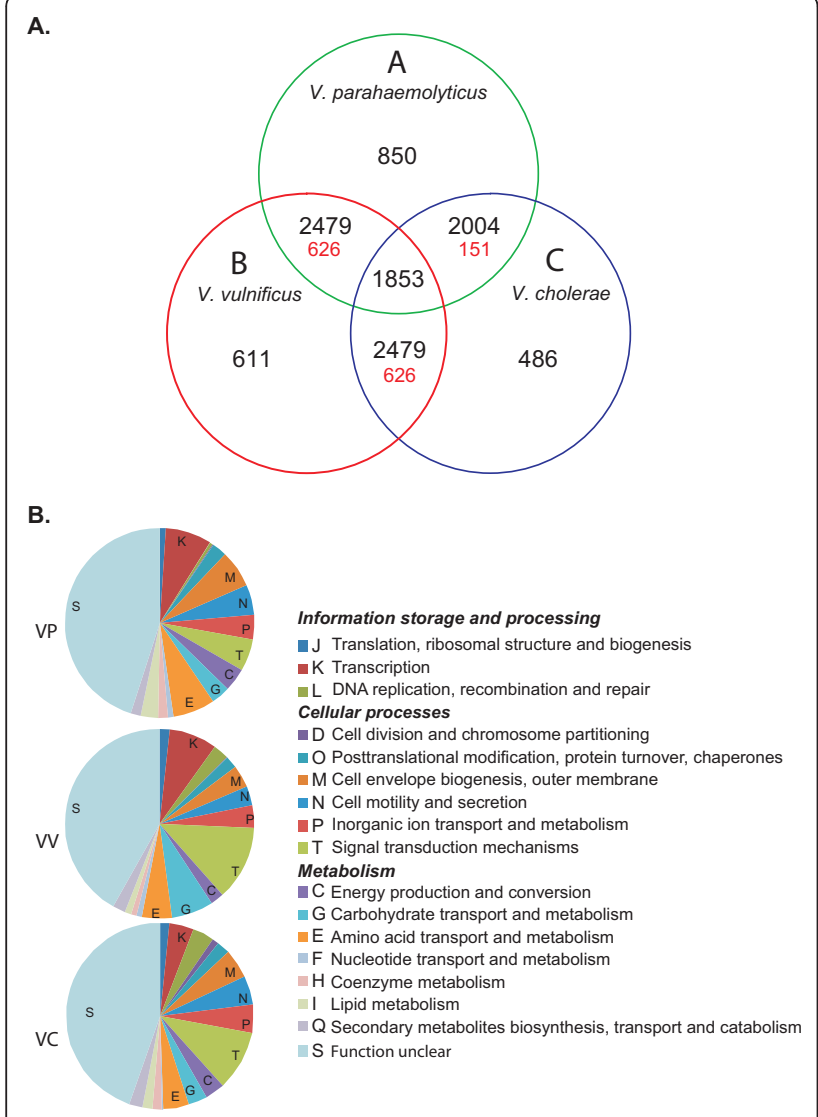

Figure 6 Species-specific core genes of $V$. parahaemolyticus, $V$. cholerae, and V. vulnificus. Left panel: Each circle represents core genes extracted from selected strains of each species. The Venn diagrams show the number of shared proteins (black) and unique proteins (red) within a particular relationship for all three species. Right panel: Pie chart of species-specific genes. Functional categories are indicated with various colors and letters.

pathways, which indicates that they have different requirements for transporting various ions, nutrients, and other metabolites across their outer membranes. In addition, each species had unique two-component regulatory systems and chemotaxis genes, which indicate that they have specific signal pathways that respond to various environmental stimuli.

The three species also differ in their outer membrane structure and virulence genes (e.g. they had different sets of genes for surface polysaccharide biosynthesis). Furthermore, $V$. vulnificus has a unique set of genes for FLP pilus synthesis, and toxigenic strains of $V$. cholerae have genes for toxin co-regulated pilus synthesis, a wellstudied virulence factor [27] (the ctx $\varnothing$ phage is not included as containing genes specific for toxigenic $V$. cholerae because it is not present in strain M66-2). V. parahaemolyticus possesses genes encoding two unique flagella, in addition to the genes required for the biosynthesis of flagella possessed by all three species. $V$. cholerae also has two different T3SS-containing islands [7]. T3SS has been reported to be closely related to T3SSII in $V$. parahaemolyticus and to be present in nonO1, non-O139 strains of $V$. cholerae [30,31], but we did not detect it in the toxigenic strains of $V$. cholerae. However, the possession of T3SS by $V$. parahaemolyticus and non-O1, non-O139 strains of $V$. cholerae suggests that this transport system is required for colonization of their unknown environmental hosts and reservoirs.

\section{Evolution of $V$. parahaemolyticus}

In order to advance our understanding of the relationships between the $V$. parahaemolyticus strains we characterized, a set of 924 single-copy genes present in all six strains (plus an outlier of $V$. vulnificus CMCP6), taken from the analysis of three pathogenic Vibrios, was compiled, and a nucleotide maximum-likelihood tree was inferred for the concatenated set of 924 genes (Additional file 3). Our expectation that a resolved phylogeny supported by the majority of genes would be found and interpreted as an estimate of the strains' phylogenies was not met, a result similar to that of Boyd et al 2008 [9] who also found that all of the pandemic strains were intermixed with pre-pandemic strains, but used just three genes in their analysis. Inspection of the aligned genomes with the SNPs marked in each one (Figure 5) revealed that the SNPs were often clustered, possibly indicative of recombination events. Recombination events involving multiple SNPs would distort the branch lengths and provide a poor estimate of the phylogeny. In retrospect, this result might have been expected because we have shown that recombination events can involve a large number of variable nucleotides in numerous genes and each one of those events will distort the distance measure used for phylogenetic trees that assume all mutations are independently acquired. In the sequenced strains, the single recombination event that converted the serotype and replaced the neighboring $90 \mathrm{~kb}$ contained 14 times the total number of variable nucleotides observed in the rest of the genome (Additional file 2, Figure 5 circle 5). Thus, if only one of the 110 genes from this region were included with the rest of the genes in the genome to calculate a tree, that one gene, on average, would contribute $11 \%$ of the total variation. In order to calculate accurate trees, each recombination event and independent mutation event must be given equal weight.

\section{Conclusion}

This study helps to improve our understanding of how $V$. parahaemolyticus evolved during a pandemic. The results of our multiple genome analyses are consistent 
with the idea that pandemic strains of $V$. parahaemolyticus evolved from pre-pandemic strains by numerous deletions and acquisitions of genetic material. Pandemic strains differ from pre-pandemic strains mostly in mobile genetic elements and the structure of the pathogenicity islands. Serotype conversion to O4:K68 was likely due to a recombination event involving a region much larger than the $\mathrm{O}$-antigen- and $\mathrm{K}$-antigen-encoding gene clusters. In addition, this study revealed that $(t d h+t r h-)$ and $(t d h-t r h+)$ strains not only have different toxin genes, but also differ in the structures and locations of their pathogenicity islands.

Lateral gene transfer seems to be the major force shaping the virulence of $V$. parahaemolyticus, as evidenced by the diversity in the locations and nucleotide sequences of the virulence factor-encoding genes. In addition, previous studies had shown that insertion sequences in $V$. parahaemolyticus could change the genome structure and result in the loss of a major virulence factor [32,33]. However, the pandemic strains we studied are almost monomorphic (except for pathogenicity islands and mobile elements) and the outbreaks in Asia and South America are closely related. During the pandemic's later development, pandemic strains with the O3:K6 serotype (and its serovariants) were no longer prevalent in India, where the pandemic originated (Nair, personal observations). Instead, massive outbreaks were reported in Chile. This observation suggests that the virulent clone that spread to South America during the pandemic's early stage has persisted in that area and continues to cause outbreaks. There was no loss of known virulence genes in the later stage pandemic (K5030) isolate from south Asia; therefore, its virulence status needs further evaluation.

Genetic changes in the etiologic agent may not be the only factor leading to $V$. parahaemolyticus-mediated pandemics (e.g. optimal environmental conditions may enable pandemic strains to flourish in their reservoirs). For example, an outbreak of foodborne diarrheal disease caused by $V$. parahaemolyticus was reported on an Alaskan cruise ship during 2004, and the source of the infection was $V$. parahaemolyticus-contaminated oysters harvested following warm weather in Alaska, where $V$. parahaemolyticus had not been previously isolated [34]. In addition, although there are major genetic differences between pre-pandemic and pandemic strains of $V$. parahaemolyticus, and they may, to some extent, contribute to the pandemic strains' increased virulence, a pathogenicity island (which contains $t d h$ and T3SS) is also present in the pre-pandemic strain AQ3810. Thus, it is likely that the recent $V$. parahaemolyticus-mediated pandemic resulted from the convergence of genetic changes in the etiologic agent and the presence of optimal conditions for survival and growth in its natural reservoirs.

\section{Materials and methods}

\section{Strain isolation and verification}

The $V$. parahaemolyticus strains were isolated on TCBS (thiosulfate, citrate, bile salts, and sucrose) agar medium followed by their presumptive identification with a multitest medium [35]. The strains' identities were confirmed by a species-specific tox $R$ assay [29], a commercially available $V$. parahaemolyticus antiserum kit (Toshiba Kagaku Kogyo Co., Ltd., Tokyo, Japan) was employed for serological typing, and $t d h$ and trh were identified by PCR [36]. Strains were cultured overnight in Luria-Bertani broth, and DNA was obtained by lysing the bacteria with proteinase $\mathrm{K}$ followed by DNA extraction and purification with a Qiagen Maxi Kit (Valencia, CA).

\section{Genome sequencing}

The genomes of $V$. parahaemolyticus strains AN5034, AQ4037, K5030, and Peru466 were sequenced by the Sanger whole-genome random shotgun method [37]. Briefly, one small insert plasmid library (3-4 kb) and one medium insert plasmid library (10-12 kb) were constructed by random nebulization and cloning of genomic DNA. During the initial random-sequencing phase, 8fold sequence coverage was achieved with the smalland medium-size libraries sequenced to yield 5-fold and 3 -fold coverage, respectively. The sequences were assembled using the Celera Assembler [38], and ordered scaffolds were generated by using NUCMER [39] to align the contigs to the genome of $V$. parahaemolyticus RIMD2210633, followed by hierarchical scaffolding with BAMBUS [40]. The contig N50 was determined as described [41].

An initial set of open-reading frames (ORFs) was identified using GLIMMER [42], and ORFs shorter than 90 bp (and some with overlaps) were eliminated. A region containing the likely origin of replication was identified, and base pair 1 was designated adjacent to the $d n a A$ gene located in that region [43]. The ORFs were searched against a nonredundant protein database, and the ORF predictions and gene family identifications were done as previously described [27]. Two sets of hidden Markov models (HMMs) were used to determine ORFs membership in families and super families. These included 721 HMMs from Pfam v22.0 and 631 HMMs from the TIGR ortholog resource. A transmembrane hidden Markov model (TMHMM) [44] was used to identify membrane-spanning domains in proteins, and putative functional role categories were assigned internally as previously described [45].

The nucleotide sequences and the corresponding automated annotations for the genomes of V. parahaemolyticus strains AN5034, AQ4037, K5030, and Peru466 were submitted to NCBI, with accession 
numbers [GenBank:ACFO00000000, Genbank: ACFN00000000, Genbank:ACKB00000000, and Genbank:ACFM00000000], respectively.

\section{Comparative genomics}

The database and cut-offs mentioned above were used, as previously described [37], to (i) produce an ortholog match table, (ii) construct a Venn diagram, and (iii) bin the relationships within the Venn diagram. Synteny plots using PROMER [39] were computed as previously described [37]. SNPs were identified by mapping chromosomal contigs to the complete reference genome of RIMD2210633 using NUCMER [39] with default setting and displayed using the SHOW-SNPS tool with the -C (do not report SNPs from alignments with an ambiguous mapping) and -I (do not report indels) options. SHOW-SNPS is part of the MUMMER 3 distribution http://mummer.sourceforge.net/. DNA maximum likelihood trees were created (using PAUP* 4.0b) for each of the 924 entries in the above mentioned ortholog table that had orthologs for $V$. vulnificus CMCP6 and the six $V$. parahaemolyticus strains. In order to ensure proper alignment of the coding regions, the trees were based on DNA alignments back-aligned from the proteins' alignments.

\section{Additional material}

Additional file 1: Vibrio parahaemolyticus Ortholog Match Table.

BLAST-based ortholog Match Table of $V$. parahaemolyticus strains

Additional file 2: Vibrio parahaemolyticus Single Nucleotide

Polymorphisms. Single Nucleotide Polymorphisms of $V$.

parahaemolyticus genomes relative to reference strain RIMD2210633

Additional file 3: Relationships of $V$. parahaemolyticus strains. DNA

Maximum Likelihood tree based on 924 orthologs of the six $V$. parahaemolyticus strains. V. vulnificus strain CMCP6 was used as an outlier.

\section{Acknowledgements}

We are grateful to the J. Craig Venter Institute's sequencing, bioinformatics and IT departments for supporting the infrastructure required to determine the genomes' sequences and annotation. We especially thank A. Scott Durkin for contributing to the genomes' annotation. We also thank Arnold S Kreger and Stephanie Zafonte for reading and editing the manuscript. This project was supported by the (i) National Institute of Allergy and Infectious Diseases (National Institutes of Health, Department of Health and Human Services), under contract number N01-Al-30071, and (ii) University of Maryland Clinical Research Unit of the Food and Waterborne Diseases Integrated Research Network (funded by the National Institute of Allergy and Infectious Diseases), under contract number N01-Al-40014.

\footnotetext{
Author details

${ }^{1}$ Emerging Pathogens Institute, University of Florida, Gainesville, FL, USA. ${ }^{2}$ Department of Pathology, University of Florida, Gainesville, FL, USA. ${ }^{3}$ School of Medicine, University of Maryland, Baltimore, MD, USA. ${ }^{4}$ Instituto de Investigación Nutricional, Peru. ${ }^{5}$ National Institute of Cholera and Enteric Diseases, Kolkata, India. ${ }^{6}$ Kyoto University, Kyoto, Japan. ${ }^{7}$ The J. Craig Venter Institute, Rockville, MD, USA.
}

\section{Authors' contributions}

OCS, RH, GBN, AIG, MN, and DEF conceived and organized the project; GBN, $A I G$, and MN provided strains; OCS and YC isolated DNA; DEF organized the sequencing studies; DEF, JHB, and $Y C$ analyzed the genomic data; and $Y C$, $\mathrm{DEF}, \mathrm{JHB}$, and OCS prepared the manuscript. All authors read and approved the final manuscript.

\section{Competing interests}

The authors declare that they have no competing interests.

Received: 21 December 2010 Accepted: 6 June 2011 Published: 6 June 2011

\section{References}

1. Fujino L, Okuno Y, Nakada D, Aoyama A, Fukai $K$, Mukai T, Uebo T: On the bacteriological examination of shirasu food poisoning. Med I Osaka Univ 1953, 4:299-304.

2. Nair GB, Ramamurthy T, Bhattacharya SK, Dutta B, Takeda Y, Sack DA: Global dissemination of Vibrio parahaemolyticus serotype O3:K6 and its serovariants. Clin Microbiol Rev 2007, 20(1):39-48

3. Nair GB, Hormazabal JC: The Vibrio parahaemolyticus pandemic. Rev Chilena Infectol 2005, 22(2):125-130.

4. Chowdhury NR, Chakraborty S, Ramamurthy T, Nishibuchi M, Yamasaki S, Takeda Y, Nair GB: Molecular evidence of clonal Vibrio parahaemolyticus pandemic strains. Emerg Infect Dis 2000, 6(6):631-636.

5. Chowdhury NR, Stine OC, Morris JG, Nair GB: Assessment of evolution of pandemic Vibrio parahaemolyticus by multilocus sequence typing. I Clin Microbiol 2004, 42(3):1280-1282.

6. Nishibuchi M, Taniguchi T, Misawa T, Khaeomanee-lam V, Honda T, Miwatani T: Cloning and nucleotide sequence of the gene (trh) encoding the hemolysin related to the thermostable direct hemolysin of Vibrio parahaemolyticus. Infect Immun 1989, 57(9):2691-2697.

7. Makino K, Oshima K, Kurokawa K, Yokoyama K, Uda T, Tagomori K, lijima Y, Najima M, Nakano M, Yamashita A, et al: Genome sequence of Vibrio parahaemolyticus: a pathogenic mechanism distinct from that of $\mathrm{V}$ cholerae. Lancet 2003, 361(9359):743-749.

8. Park KS, Ono T, Rokuda M, Jang MH, Okada K, lida T, Honda T: Functional characterization of two type III secretion systems of Vibrio parahaemolyticus. Infect Immun 2004, 72(11):6659-6665.

9. Boyd EF, Cohen AL, Naughton LM, Ussery DW, Binnewies TT, Stine OC, Parent MA: Molecular analysis of the emergence of pandemic Vibrio parahaemolyticus. BMC Microbiol 2008, 8:110.

10. Gil Al, Miranda H, Lanata CF, Prada A, Hall ER, Barreno CM, Nusrin S, Bhuiyan NA, Sack DA, Nair GB: O3:K6 serotype of Vibrio parahaemolyticus identical to the global pandemic clone associated with diarrhea in Peru. Int J Infect Dis 2007, 11(4):324-328.

11. Fouts DE: Phage_Finder: automated identification and classification of prophage regions in complete bacterial genome sequences. Nucleic Acids Res 2006, 34(20):5839-5851.

12. Nasu H, lida T, Sugahara T, Yamaichi Y, Park KS, Yokoyama K, Makino K Shinagawa $\mathrm{H}$, Honda T: A filamentous phage associated with recent pandemic Vibrio parahaemolyticus O3:K6 strains. J Clin Microbiol 2000, 38(6):2156-2161.

13. Okada N, lida T, Park KS, Goto N, Yasunaga T, Hiyoshi H, Matsuda S, Kodama T, Honda T: Identification and characterization of a novel type III secretion system in trh-positive Vibrio parahaemolyticus strain TH3996 reveal genetic lineage and diversity of pathogenic machinery beyond the species level. Infect Immun 2009, 77(2):904-913.

14. Nishibuchi M, Kaper JB: Thermostable direct hemolysin gene of Vibrio parahaemolyticus: a virulence gene acquired by a marine bacterium. Infect Immun 1995, 63(6):2093-2099.

15. Shirai $\mathrm{H}$, Ito $\mathrm{H}$, Hirayama $T$, Nakamoto $\mathrm{Y}$, Nakabayashi $\mathrm{N}$, Kumagai $\mathrm{K}$, Takeda Y, Nishibuchi M: Molecular epidemiologic evidence for association of thermostable direct hemolysin (TDH) and TDH-related hemolysin of Vibrio parahaemolyticus with gastroenteritis. Infect Immun 1990, 58(11):3568-3573.

16. Kodama T, Hiyoshi H, Gotoh K, Akeda Y, Matsuda S, Park KS, Cantarelli W, lida T, Honda T: Identification of two translocon proteins of Vibrio parahaemolyticus type III secretion system 2. Infect Immun 2008, 76(9):4282-4289.

17. Kodama T, Rokuda M, Park KS, Cantarelli W, Matsuda S, lida T, Honda T: Identification and characterization of VopT, a novel ADP- 
ribosyltransferase effector protein secreted via the Vibrio parahaemolyticus type III secretion system 2. Cell Microbiol 2007, 9(11):2598-2609

18. Ono T, Park KS, Ueta M, lida T, Honda T: Identification of proteins secreted via Vibrio parahaemolyticus type III secretion system 1. Infect Immun 2006, 74(2):1032-1042.

19. Chen Y, Dai J, Morris JG, Johnson JA: Genetic analysis of the capsule polysaccharide ( $\mathrm{K}$ antigen) and exopolysaccharide genes in pandemic Vibrio parahaemolyticus O3:K6. 2010, 10:274.

20. Chen Y, Dai J, Johnson JA, Morris JG: Genetic determinant of the capsule polysaccharide ( $\mathrm{K}$ antigen) in pandemic Vibrio parahaemolyticus 03:K6. 2009.

21. Okura M, Osawa R, Tokunaga A, Morita M, Arakawa E, Watanabe H: Genetic analyses of the putative $\mathrm{O}$ and $\mathrm{K}$ antigen gene clusters of pandemic Vibrio parahaemolyticus. Microbiol Immunol 2008, 52(5):251-264.

22. Zhou X, He X, Liang J, Li A, Xu T, Kieser T, Helmann JD, Deng Z: A novel DNA modification by sulphur. Mol Microbiol 2005, 57(5):1428-1438.

23. Zhou X, Deng Z, Firmin JL, Hopwood DA, Kieser T: Site-specific degradation of Streptomyces lividans DNA during electrophoresis in buffers contaminated with ferrous iron. Nucleic Acids Res 1988, 16(10):4341-4352.

24. Wang L, Chen S, Vergin KL, Giovannoni SJ, Chan SW, Demott MS, Taghizadeh K, Cordero OX, Cutler M, Timberlake S, et al: DNA phosphorothioation is widespread and quantized in bacterial genomes. Proc Natl Acad Sci USA 2011.

25. Chun J, Grim CJ, Hasan NA, Lee JH, Choi SY, Haley BJ, Taviani E, Jeon YS, Kim DW, Lee JH, et al: Comparative genomics reveals mechanism for short-term and long-term clonal transitions in pandemic Vibrio cholerae. Proc Natl Acad Sci USA 2009, 106(36):15442-15447.

26. Feng $L$, Reeves PR, Lan R, Ren Y, Gao C, Zhou Z, Ren Y, Cheng J, Wang W, Wang J, et al: A recalibrated molecular clock and independent origins for the cholera pandemic clones. PLoS One 2008, 3(12):e4053.

27. Heidelberg JF, Eisen JA, Nelson WC, Clayton RA, Gwinn ML, Dodson RJ, Haft DH, Hickey EK, Peterson JD, Umayam L, et al: DNA sequence of both chromosomes of the cholera pathogen Vibrio cholerae. Nature 2000 406(6795):477-483.

28. Chen CY, Wu KM, Chang YC, Chang CH, Tsai HC, Liao TL, Liu YM, Chen HJ, Shen $A B$, Li JC, et al: Comparative genome analysis of Vibrio vulnificus, a marine pathogen. Genome Res 2003, 13(12):2577-2587.

29. Kim YB, Okuda J, Matsumoto C, Takahashi N, Hashimoto S, Nishibuchi M: Identification of Vibrio parahaemolyticus strains at the species level by PCR targeted to the toxR gene. J Clin Microbiol 1999, 37(4):1173-1177.

30. Chen Y, Johnson JA, Pusch GD, Morris JG Jr, Stine OC: The genome of non-01 Vibrio cholerae NRT36S demonstrates the presence of pathogenic mechanisms that are distinct from those of 01 Vibrio cholerae. Infect Immun 2007, 75(5):2645-2647.

31. Dziejman M, Serruto D, Tam VC, Sturtevant D, Diraphat P, Faruque SM, Rahman MH, Heidelberg JF, Decker J, Li L, et al: Genomic characterization of non-01, non-0139 Vibrio cholerae reveals genes for a type III secretion system. Proc Natl Acad Sci USA 2005, 102(9):3465-3470.

32. Kamruzzaman M, Bhoopong P, Vuddhakul V, Nishibuchi M: Detection of a functional insertion sequence responsible for deletion of the thermostable direct hemolysin gene $(t d h)$ in Vibrio parahaemolyticus. Gene 2008, 421(1-2):67-73.

33. Kamruzzaman M, Nishibuchi M: Detection and characterization of a functional insertion sequence, ISVpa2, in Vibrio parahaemolyticus. Gene 2008, 409(1-2):92-99.

34. The Great Convergence: People, Animals, and the Environment. [http:// www.cdc.gov/nczved/framework/features/convergence.html].

35. Bag PK, Nandi S, Bhadra RK, Ramamurthy T, Bhattacharya SK, Nishibuchi M, Hamabata T, Yamasaki S, Takeda Y, Nair GB: Clonal diversity among recently emerged strains of Vibrio parahaemolyticus 03:K6 associated with pandemic spread. J Clin Microbiol 1999, 37(7):2354-2357.

36. Tada J, Ohashi T, Nishimura N, Shirasaki Y, Ozaki H, Fukushima S, Takano J, Nishibuchi M, Takeda Y: Detection of the thermostable direct hemolysin gene $(t d h)$ and the thermostable direct hemolysin-related hemolysin gene (trh) of Vibrio parahaemolyticus by polymerase chain reaction. $\mathrm{Mol}$ Cell Probes 1992, 6(6):477-487.

37. Fouts DE, Mongodin EF, Mandrell RE, Miller WG, Rasko DA, Ravel J, Brinkac LM, DeBoy RT, Parker CT, Daugherty SC, et al: Major structural differences and novel potential virulence mechanisms from the genomes of multiple Campylobacter species. PLoS Biol 2005, 3(1):e15.

38. Myers EW, Sutton GG, Delcher AL, Dew IM, Fasulo DP, Flanigan MJ, Kravitz SA, Mobarry CM, Reinert KH, Remington KA, et al: A whole-genome assembly of Drosophila. Science 2000, 287(5461):2196-2204.

39. Delcher AL, Phillippy A, Carlton J, Salzberg SL: Fast algorithms for largescale genome alignment and comparison. Nucleic Acids Research 2002 30(11):2478-2483.

40. Popovic T, Bopp C, Olsvik O, Wachsmuth K: Epidemiologic application of a standardized ribotype scheme for Vibrio cholerae 01. J Clin Microbiol 1993, 31(9):2474-2482.

41. Miller JR, Koren S, Sutton G: Assembly algorithms for next-generation sequencing data. Genomics 2010, 95(6):315-327.

42. Delcher AL, Harmon D, Kasif S, White O, Salzberg SL: Improved microbial gene identification with GLIMMER. Nucleic Acids Research 1999, 27(23):4636-4641.

43. Bramhill D, Kornberg A: Duplex opening by dnaA protein at novel sequences in initiation of replication at the origin of the $E$. coli chromosome. Cell 1988, 52(5):743-755.

44. Krogh A, Larsson B, von Heijne G, Sonnhammer EL: Predicting transmembrane protein topology with a hidden Markov model: application to complete genomes. J Mol Biol 2001, 305(3):567-580

45. Fleischmann RD, Adams MD, White O, Clayton RA, Kirkness EF, Kerlavage AR, Bult CJ, Tomb JF, Dougherty BA, Merrick JM, et al: Wholegenome random sequencing and assembly of Haemophilus influenzae Rd. Science 1995, 269(5223):496-512.

doi:10.1186/1471-2164-12-294

Cite this article as: Chen et al:: Comparative genomic analysis of Vibrio parahaemolyticus: serotype conversion and virulence. BMC Genomics 2011 12:294

\section{Submit your next manuscript to BioMed Central and take full advantage of:}

- Convenient online submission

- Thorough peer review

- No space constraints or color figure charges

- Immediate publication on acceptance

- Inclusion in PubMed, CAS, Scopus and Google Scholar

- Research which is freely available for redistribution

Submit your manuscript at www.biomedcentral.com/submit
Ciomed Central 